PROCEEDINGS OF THE

AMERICAN MATHEMATICAL SOCIETY

Volume 128, Number 10, Pages 2973-2979

S 0002-9939(00)05380-6

Article electronically published on March 3, 2000

\title{
ON INTERPOLATION FAMILIES OF WAVELET SETS
}

\author{
QING GU
}

(Communicated by Dale Alspach)

\begin{abstract}
The question of which groups are isomorphic to groups of interpolation maps for interpolation families of wavelet sets was raised by Dai and Larson. In this article it is shown that any finite group is isomorphic to a group of interpolation maps for some interpolation family of wavelet sets.
\end{abstract}

A dyadic orthonormal (or orthogonal) wavelet is a function $\psi \in L^{2}(\mathbb{R})$ (Lebesgue measure), with the property that the set

$$
\left\{2^{\frac{n}{2}} \psi\left(2^{n} t-l\right): n, l \in \mathbb{Z}\right\}
$$

is an orthonormal basis for $L^{2}(\mathbb{R})$.

For certain measurable sets $E \subseteq \mathbb{R}$ the normalized characteristic function $\frac{1}{\sqrt{2 \pi}} \chi_{E}$ is the Fourier transform of such a wavelet. There are several characterizations of such sets. In DL] they are called wavelet sets, and the corresponding wavelet is called an s-elementary wavelet. In [FW], HWW1, [HWW2] they are the support sets of MFS (minimal frequency supported) wavelets.

In [DL], a method of operator-theoretic interpolation between certain special pairs of wavelets, and between single wavelets and special families of them is developed. The interpolation pairs of wavelet sets are extensively discussed and the method yields a new construction of a class of wavelets due to Meyer. An interpolation pair is the simplest case of an interpolation family (see definitions below). Affiliated to an interpolation family is a group (under composition) of measure preserving transformations of $\mathbb{R}$ called the interpolation maps. Problem $\mathbf{E}$ in $[\mathrm{DL}]$ asked for a characterization of all groups which are isomorphic to a group of interpolation maps for some interpolation family of wavelet sets. They showed, by example, that the cyclic groups $\mathbb{Z}_{2}$ and $\mathbb{Z}_{3}$ of order 2 and 3 could be realized in this way, but they proved no cases of other groups. The purpose of this paper is to prove that every finite group can be realized in this way.

Before introducing the characterization of wavelet sets, we will need some more terminology. In [DL, two measurable sets $E, F$ are called translation congruent modulo $2 \pi$ if there is a measurable bijection $\phi: E \rightarrow F$ such that $\phi(s)-s$ is an integral multiple of $2 \pi$ for each $s \in E$. Analogously, two measurable sets $G, H$ are called dilation congruent modulo 2 if there is a measurable bijection $\tau: G \rightarrow H$ such that for each $s \in G$ there is an integer $n$ depending on $s$, such that $\tau(s)=2^{n} s$. We note in passing that if $E_{i}$ and $F_{i}$ are measurable subsets of $\mathbb{R}$ such that $E_{i}$ and

Received by the editors July 7, 1998 and, in revised form, November 24, 1998.

2000 Mathematics Subject Classification. Primary 42C40.

Key words and phrases. Interpolation family of wavelet sets, wavelet.

(C)2000 American Mathematical Society 
$F_{i}$ are translation congruent modulo $2 \pi$ for each $i \in\{1,2, . ., n\}$, and $E_{i} \cap E_{j}=$ $\emptyset, F_{i} \cap F_{j}=\emptyset$ for any distinct $i, j \in\{1,2, . ., n\}$, then $\bigcup_{i=1}^{i=n} E_{i}$ and $\bigcup_{i=1}^{i=n} F_{i}$ are translation congruent modulo $2 \pi$. Likewise, if $E_{i}$ and $F_{i}$ are measurable subsets of $\mathbb{R}$ such that $E_{i}$ and $F_{i}$ are dilation congruent modulo 2 for each $i \in\{1,2, . ., n\}$, and $E_{i} \cap E_{j}=\emptyset, F_{i} \cap F_{j}=\emptyset$ for any distinct $i, j \in\{1,2, . ., n\}$, then $\bigcup_{i=1}^{i=n} E_{i}$ and $\bigcup_{i=1}^{i=n} F_{i}$ are dilation congruent modulo 2 .

A measurable subset $G \subseteq \mathbb{R}$ is called a 2-dilation generator of a partition of $\mathbb{R}$ if the sets $2^{n} G:=\left\{2^{n} s: s \in G\right\}, n \in \mathbb{Z}$, are disjoint and $\mathbb{R} \backslash \bigcup_{n} 2^{n} G$ is a null set. Likewise a measurable subset $H \subseteq \mathbb{R}$ is called a $2 \pi$-translation generator of a partition of $\mathbb{R}$ if the sets $H+2 n \pi:=\{s+2 n \pi: s \in H\}, n \in \mathbb{Z}$, are disjoint and $\mathbb{R} \backslash \bigcup_{n}(H+2 n \pi)$ is a null set.

We have the following characterization of wavelet sets (see Lemma 4.3 in $[\mathrm{DL}$ ): Let $E \subseteq \mathbb{R}$ be a measurable set. Then $E$ is a wavelet set if and only if $E$ is both a 2-dilation generator of a partition (modulo null sets) of $\mathbb{R}$ and a $2 \pi$-translation generator of a partition (modulo null sets) of $\mathbb{R}$. Equivalently $E$ is a wavelet set if and only if $E$ is both translation congruent to $[0,2 \pi)$ modulo $2 \pi$ and dilation congruent to $[-2 \pi,-\pi) \cup[\pi, 2 \pi)$ modulo 2 .

So the simplest wavelet set is $[-2 \pi,-\pi) \cup[\pi, 2 \pi)$.

Again, following [DL, let $E$ and $F$ be wavelet sets and let $\sigma: E \rightarrow F$ be the $1-1$, onto map implementing the $2 \pi$-translation congruence. Then we can extend $\sigma$ to a 1-1 map (modulo a null set) of $\mathbb{R}$ onto $\mathbb{R}$ by defining $\sigma(0)=0$, and

$$
\sigma(s)=2^{n} \sigma\left(2^{-n} s\right)
$$

for $s \in 2^{n} E, n \in \mathbb{Z}$. We adopt the notation $\sigma_{E}^{F}$ for this map, and call it the interpolation map for the ordered pair of wavelet sets $(E, F)$. It is shown in [DL] that $\sigma_{E}^{F}$ is a measure-preserving transformation from $\mathbb{R}$ to $\mathbb{R}$.

A measurable function $f: \mathbb{R} \rightarrow \mathbb{R}$ is called 2-homogeneous if $f(2 s)=2 f(s)$ for a.e. $s \in \mathbb{R}$. Clearly, $\sigma_{E}^{F}$ defined above is such a 2-homogeneous function. As observed in [DL], any 2-homogeneous function is completely determined a.e. by its values on any subset of $\mathbb{R}$ which is a 2-dilation generator of a partition of $\mathbb{R}$ (in particular, a wavelet set). So $\sigma_{E}^{F}$ is the unique 2-homogeneous extension of the $2 \pi$-translation congruence $E \rightarrow F$.

We say that a pair of wavelet sets $(E, F)$ is an interpolation pair if $\left(\sigma_{E}^{F}\right) \circ\left(\sigma_{E}^{F}\right)$ is the identity map on $\mathbb{R}$ a.e. In general, an interpolation family of wavelet sets based at $E_{0}$ is a family $\mathcal{E}$ of wavelet sets with $E_{0} \in \mathcal{E}$ such that $\left\{\sigma_{E_{0}}^{E}: E \in \mathcal{E}\right\}$ is a group (of measure-preserving transformations of $\mathbb{R}$ ) under composition.

In the construction of interpolation families of wavelet sets discussed below, one theorem from Speegle's paper [S] concerning path-connectedness of the s-elementary wavelets plays an important role.

In $\left[\right.$ S] measurable maps $\tilde{\tau}: \mathbb{R} \rightarrow[-2 \pi,-\pi) \cup[\pi, 2 \pi)$ and $\tilde{d}: \mathbb{R}_{0}=(\mathbb{R} \backslash\{0\}) \rightarrow$ $[-2 \pi,-\pi) \cup[\pi, 2 \pi)$ are defined by $\tilde{\tau}(x)=x+2 \pi m(x), \tilde{d}(x)=2^{n(x)} x$, where $m$ and $n$ are the unique integers which map $x$ into $[-2 \pi,-\pi) \cup[\pi, 2 \pi)$ under translation by $2 \pi m$ and dilation by $2^{n}$ respectively. Thus $W$ is a wavelet set if and only if $\left.\tilde{d}\right|_{W}$ and $\left.\tilde{\tau}\right|_{W}$ are bijections (modulo null sets). If $\left.\tilde{d}\right|_{W}$ and $\left.\tilde{\tau}\right|_{W}$ are injections modulo null sets, then $W$ is called a sub-wavelet set.

For a subwavelet set $A$ we define two subsets of $[-2 \pi,-\pi) \cup[\pi, 2 \pi)$ by $A^{d}=\tilde{d}(A)$ and $A^{\tau}=\tilde{\tau}(A)$. 
Theorem $1([\mathrm{~S}])$. Let $A$ be a sub-wavelet set. Suppose

(1) there is an $\epsilon>0$ such that $A^{\tau} \subset[-2 \pi+\epsilon,-\pi) \cup[\pi, 2 \pi-\epsilon)$ and

(2) $S:=([-2 \pi,-\pi) \cup[\pi, 2 \pi)) \backslash A^{d}$ has non-empty interior.

Then, there is a wavelet set $W \supset A$.

Example A.9 in $[\mathrm{DL}$ is an example of an interpolation map $\sigma$ between two wavelet sets $E$ and $F$ for which $\sigma^{3}$ is the identity map, and also for which $\sigma^{2}$ is a $2 \pi$-congruence on the initial wavelet set $E$. That is, $\left(\sigma^{2}(x)-x\right) / 2 \pi \in \mathbb{Z}$ for all $x \in E$. In this example

$$
\begin{gathered}
A=\left[-\pi,-\frac{\pi}{2}\right) \cup\left[\frac{7 \pi}{2}, \frac{31 \pi}{8}\right), \\
B=\left[-\frac{\pi}{4},-\frac{\pi}{8}\right) \cup\left[7 \pi, \frac{31 \pi}{4}\right)
\end{gathered}
$$

and $E=A \cup C$ and $F=B \cup C$ for a certain measurable set $C$. Therefore if we denote $\sigma^{2}(E)$ as $G$, then it can be proved that $G$ is a wavelet set. Moreover it can be checked that $\left\{\sigma_{E}^{F}, \sigma_{E}^{G}, \sigma_{E}^{E}\right\}$ is a group isomorphic to $\mathbb{Z}_{3}$ and so $\{E, F, G\}$ is an interpolation family of wavelet sets based at $E$.

The construction in this paper is a generalization of this example.

Theorem 2. For any $k \in \mathbb{N}$ with $k \geq 2$ there exists an interpolation family $\mathcal{E}$ of wavelet sets based at $E_{0} \in \mathcal{E}$ such that $\left\{\sigma_{E_{0}}^{E}: E \in \mathcal{E}\right\}$ is isomorphic to the symmetric group of degree $k$.

We need the following lemmas to prove the theorem. We use $m(\cdot)$ to denote Lebesgue measure.

Lemma 3. Let $k \geq 2$ be an integer. Let $A=\left[-\pi,-\frac{\pi}{2}\right) \cup\left[\frac{2^{k}-1}{2} \pi, \frac{2^{2 k-1}-1}{2^{k}} \pi\right)$, and denote $A_{1}=\left[-\pi,-\frac{\pi}{2}\right)$, and $A_{i}=\left[\frac{2^{k+i-2}-1}{2^{i-1}} \pi, \frac{2^{k+i-1}-1}{2^{i}} \pi\right)$ for $2 \leq i \leq k$. Then the following hold:

(i) $A=\bigcup_{i=1}^{k} A_{i}$ and $m\left(A_{i}\right)=\frac{1}{2^{i}} \pi$ for all $i \in\{1, \ldots, k\}$. Moreover for all $i \neq j$, with $i, j \in\{1, \ldots, k\}, A_{i} \cap A_{j}=\emptyset$.

(ii) For all $i \neq j$, with $i, j \in\{1, \ldots, k\}$, the number $l=2^{j-i}$ is the unique positive real number such that $m\left(A_{i}\right)=m\left(l A_{j}\right)$. Moreover the sets $A_{i}$ and $l A_{j}$ are $2 \pi$-translation congruent.

Proof of Lemma 3. To prove (i), note that we can write $\left[\frac{2^{k}-1}{2} \pi, \frac{2^{2 k-1}-1}{2^{k}} \pi\right)$ as $2^{k-1} \pi$ $+\left[-\frac{1}{2} \pi,-\frac{1}{2^{k}} \pi\right)$. Also we can write $A_{i}$ as $A_{i}=2^{k-1} \pi+\left[-\frac{1}{2^{i-1}} \pi,-\frac{1}{2^{i}} \pi\right)$ for $2 \leq i \leq k$. Now

$$
\left[-\frac{1}{2} \pi,-\frac{1}{2^{k}} \pi\right)=\bigcup_{i=2}^{k}\left[-\frac{1}{2^{i-1}} \pi,-\frac{1}{2^{i}} \pi\right) .
$$

This implies $A=\bigcup_{i=1}^{k} A_{i}$. It is clear that $m\left(A_{i}\right)=\frac{1}{2^{i}} \pi$ for all $i \in\{1, \ldots, k\}$.

To see that $A_{i} \cap A_{j}=\emptyset$ for any pair of distinct $i, j \in\{1, \ldots, k\}$, first we note that since $A_{1}$ is contained in $(-\infty, 0)$, we have $A_{1} \cap A_{i}=\emptyset$ for any $i \in\{2, \ldots, k\}$. Next we observe that for any distinct $i, j \in\{2, \ldots, k\}$,

$$
\left[-\frac{1}{2^{i-1}} \pi,-\frac{1}{2^{i}} \pi\right) \cap\left[-\frac{1}{2^{j-1}} \pi,-\frac{1}{2^{j}} \pi\right)=\emptyset .
$$

So by the way the $A_{i}$ 's are defined, we have $A_{i} \cap A_{j}=\emptyset$ for any distinct pair of $i, j \in\{2, \ldots, k\}$. Therefore $A_{i} \cap A_{j}=\emptyset$ for any distinct pair of $i, j \in\{1, \ldots, k\}$. 
To prove (ii), we first note that from (i) we have $m\left(A_{i}\right)=\frac{1}{2^{i}} \pi$ for all $i \in\{1, \ldots, k\}$. Since the $A_{i}$ 's are intervals, for any $l>0, m\left(l A_{i}\right)$ must be $l \cdot \frac{1}{2^{\imath}} \pi$. So for fixed $i \neq j$ the positive number $l$ which makes $m\left(A_{i}\right)=m\left(l A_{j}\right)$ must be unique. Now let $l=2^{j-i}$ and observe that $m\left(A_{i}\right)=m\left(l A_{j}\right)$.

Last of all, in order to see the $2 \pi$-translation congruence between $A_{i}$ and $l \cdot A_{j}$ we recall that $A_{1}=\left[-\pi,-\frac{\pi}{2}\right)$ and that $A_{i}$ can be written as

$$
A_{i}=2^{k-1} \pi+\left[-\frac{1}{2^{i-1}} \pi,-\frac{1}{2^{i}} \pi\right)
$$

for $2 \leq i \leq k$ as in the proof of (i). Thus the following are easily obtained:

1) For $j \neq 1, A_{1}=2^{j-1} A_{j}-2^{k+j-2} \pi$;

2) For $i \neq 1, A_{i}=2^{k-1} \pi+2^{1-i} A_{1}$;

3) For $i \neq 1, j \neq 1, i \neq j, A_{i}=2^{j-i} A_{j}-2^{k+j-i-1} \pi+2^{k-1} \pi$.

Thus all the cases are covered and in each case the $2 \pi$-translation congruence between $A_{i}$ and $2^{j-i} A_{j}$ is evident. Note that the Lebesgue measures of $A_{i}$ and $2^{j-i} A_{j}$ are clearly the same.

As usual, we denote the symmetric group of degree $k$ as $S_{k}$, where the elements are bijections $p:\{1, \ldots, k\} \rightarrow\{1, \ldots, k\}$ (permutations of $\{1, \ldots, k\}$ ) and the binary operation is functional composition. We also need the following lemma.

Lemma 4. Let $k, A, A_{1}$ and $A_{i}$ be as in Lemma 3. For each $p \in S_{k}$, let $A_{p}:=$ $\bigcup_{i=1}^{k} 2^{p(i)-i} A_{p(i)}$. Then the following hold:

(i) $\left\{A_{p}: p \in S_{k}\right\}$ consists of mutually $2 \pi$-translation and 2-dilation congruent elements.

(ii) $A_{p}$ is a sub-wavelet set and satisfies the criteria in Theorem 1 for all $p \in S_{k}$.

Proof of Lemma 4. To prove (i), note first that if $p=i d \in S_{k}$, then $A_{p}=A$. Note also that from Lemma 3 , for all $i \neq j$, with $i, j \in\{1, \ldots, k\}, A_{i} \cap A_{j}=\emptyset$. We claim that for fixed $p \in S_{k}$, and for all $i \neq j$, with $i, j \in\{1, \ldots, k\}$,

$$
\left(2^{p(i)-i} A_{p(i)}\right) \cap\left(2^{p(j)-j} A_{p(j)}\right)=\emptyset .
$$

Indeed, let $l \in\{1, \ldots, k\}$ be the unique integer such that $p(l)=1$. Then $2^{p(l)-l} A_{p(l)}$ is the only interval among the sets $2^{p(i)-i} A_{p(i)}$ such that $2^{p(l)-l} A_{p(l)} \subseteq$ $(-\infty, 0)$. Therefore $\left(2^{p(l)-l} A_{p(l)}\right) \cap\left(2^{p(j)-j} A_{p(j)}\right)=\emptyset$ for any $j \neq l$. Now for any $i \neq l$ such that $i \in\{1, \ldots, k\}$, we have $p(i) \neq 1$ and

$$
2^{p(i)-i} A_{p(i)}=2^{p(i)-i+k-1} \pi+\left[-\frac{1}{2^{i-1}} \pi,-\frac{1}{2^{i}} \pi\right) .
$$

Thus $\left(2^{p(i)-i} A_{p(i)}\right) \cap\left(2^{p(j)-j} A_{p(j)}\right)=\emptyset$ for any distinct $i, j \neq l$ with $i, j \in\{1, \ldots, k\}$. Hence for all $i \neq j$ such that $i, j \in\{1, \ldots, k\}$, we must have $\left(2^{p(i)-i} A_{p(i)}\right) \cap$ $\left(2^{p(j)-j} A_{p(j)}\right)=\emptyset$.

Now for any fixed $p \in S_{k}$ and for each $i \in\{1, \ldots, k\}$ we have

$$
m\left(2^{p(i)-i} A_{p(i)}\right)=2^{p(i)-i} \cdot \frac{1}{2^{p(i)}} \pi=\frac{1}{2^{i}} \pi=m\left(A_{i}\right) .
$$

So by Lemma 3 , the sets $A_{i}$ and $2^{p(i)-i} A_{p(i)}$ are $2 \pi$-translation congruent for any $i \in\{1, \ldots, k\}$. Since the sets $A_{i}$ are pairwise disjoint, and the sets $2^{p(i)-i} A_{p(i)}$ are also pairwise disjoint, it follows that $A$ is $2 \pi$-translation congruent to $A_{p}$ for any $p \in S_{k}$. 
Likewise, observe that $A_{p(i)}$ and $2^{p(i)-i} A_{p(i)}$ are 2-dilation congruent for any $i \in\{1, \ldots, k\}$. For any fixed $p \in S_{k}, p:\{1, \ldots, k\} \rightarrow\{1, \ldots, k\}$ is a bijection, so $\left\{A_{i}: i \in\{1, \ldots, k\}\right\}=\left\{A_{p(i)}: i \in\{1, \ldots, k\}\right\}$. Once again since the sets $A_{i}$ are pairwise disjoint, the sets $2^{p(i)-i} A_{p(i)}$ are also pairwise disjoint, it follows that $A$ is 2-dilation congruent to $A_{p}$ for any $p \in S_{k}$.

We have shown that $A$ is both $2 \pi$-translation congruent and 2-dilation congruent to $A_{p}$ for any $p \in S_{k}$. Therefore $\left\{A_{p}: p \in S_{k}\right\}$ consists of mutually $2 \pi$-translation and 2-dilation congruent elements.

To prove (ii), namely to check that $A_{p}$ is a sub-wavelet set and satisfies the criteria in Theorem 1 for all $p \in S_{k}$, it is enough to check any one of them since (i) has been established. Without loss of generality, let us look at

$$
A_{i d}=A=\left[-\pi,-\frac{\pi}{2}\right) \cup\left[\frac{2^{k}-1}{2} \pi, \frac{2^{2 k-1}-1}{2^{k}} \pi\right) .
$$

First of all, since $2\left[-\pi,-\frac{1}{2} \pi\right)=[-2 \pi,-\pi)$ we have $\tilde{d}(x)=2 x$ for $x \in\left[-\pi,-\frac{\pi}{2}\right)$. Furthermore, since $2^{-k+2}\left[\frac{2^{k}-1}{2} \pi, \frac{2^{2 k-1}-1}{2^{k}} \pi\right)=\left[\frac{2^{k}-1}{2^{k-1}} \pi, \frac{2^{2 k-1}-1}{2^{2 k-2}} \pi\right)$ which is contained in $[\pi, 2 \pi)$, it follows that $\tilde{d}(x)=2^{-k+2} x$ for $x \in\left[\frac{2^{k}-1}{2} \pi, \frac{2^{2 k-1}-1}{2^{k}} \pi\right)$. Therefore $\left.\tilde{d}\right|_{A}$ is injective. As for the injectiveness of $\left.\tilde{\tau}\right|_{A}$, recall again that $A=\bigcup_{i=1}^{k} A_{i}$ with $A_{1}=\left[-\pi,-\frac{\pi}{2}\right)$ and $A_{i}=2^{k-1} \pi+\left[-\frac{1}{2^{i-1}} \pi,-\frac{1}{2^{i}} \pi\right)$ for $2 \leq i \leq k$. Thus it is immediate that $\tilde{\tau}(x)=x+2 \pi$ for $x \in A_{1}$ and $\tilde{\tau}(x)=x+2 \pi+2^{k-1} \pi$ for $x \in A_{i}$ and $2 \leq i \leq k$. Thus $\left.\tilde{\tau}\right|_{A}$ is injective. So $A$ is a sub-wavelet set.

Since the above formulae for $\left.\tilde{d}\right|_{A}$ and $\left.\tilde{\tau}\right|_{A}$ show that

$$
\begin{gathered}
A^{\tau}=\left[\pi, 2 \pi-\frac{1}{2^{k}} \pi\right), \\
A^{d}=[-2 \pi,-\pi) \cup\left[\frac{2^{k}-1}{2^{k-1}} \pi, \frac{2^{2 k-1}-1}{2^{2 k-2}} \pi\right),
\end{gathered}
$$

$A$ satisfies the criteria in Theorem 1.

Now we are ready to prove Theorem 2 .

Proof of Theorem 2. Let $k \geq 2$ be an integer. Let $A, A_{i}$ and $A_{p}$ be the same sets as defined in Lemma 3 for each $1 \leq i \leq k$ and each $p \in S_{k}$. By Lemma $3, A_{i d}=A$ is a sub-wavelet set and satisfies the criteria in Theorem 1 . Thus there exists a measurable set $C$, such that $C \cap A=\emptyset$ and $W_{i d}:=C \cup A$ is a wavelet set. Also by Lemma $3,\left\{A_{p}: p \in S_{k}\right\}$ consists of mutually $2 \pi$-translation and 2-dilation congruent elements. Therefore, such a set $C$ satisfies $C \cap A_{p}=\emptyset$ and $W_{p}:=C \cup A_{p}$ is a wavelet set for every $p \in S_{k}$.

Let $\mathcal{E}:=\left\{W_{p}: p \in S_{k}\right\}$. We will prove that $\left\{\sigma_{W_{i d}}^{W_{p}}: W_{p} \in \mathcal{E}\right\}$ is isomorphic to the group $S_{k}$. Thus it will follow that $\mathcal{E}$ is the desired interpolation family of wavelet sets based at $E_{0}=W_{i d}$.

Denote $\sigma_{p}:=\sigma_{W_{i d}}^{W_{p}}$. Obviously $\sigma_{p}(C)=C$ and for any $x \in C, \sigma_{p}(x)=x$. Also since $\sigma_{p}$ implements the $2 \pi$-translation congruence between $W_{i d}$ and $W_{p}$, the restriction of $\sigma_{p}$ on the interval $A_{i}$ is a linear 1-1 map onto the interval $2^{p(i)-i} \cdot A_{p(i)}$ and it maps the left end point of $A_{i}$ to the left end point of $2^{p(i)-i} \cdot A_{p(i)}$ for each $i \in\{1, \ldots, k\}$ and each $p \in S_{k}$.

Therefore since $\sigma_{p}$ is 2-homogeneous, for any integer $l$, we must have $\sigma_{p}\left(2^{l} C\right)=$ $2^{l} C$ and for any $x \in 2^{l} C, \sigma_{p}(x)=x$. Also the restriction of $\sigma_{p}$ on interval $2^{l} A_{i}$ is a linear 1-1 map onto interval $2^{l} \cdot 2^{p(i)-i} \cdot A_{p(i)}$ and it maps the left-hand end point of 
$2^{l} A_{i}$ to the left-hand end point of $2^{l} \cdot 2^{p(i)-i} \cdot A_{p(i)}$ for each $i \in\{1, \ldots, k\}$ and each $p \in S_{k}$ and each integer $l$. In particular,

$$
\begin{aligned}
\left(\sigma_{p_{1}} \circ \sigma_{p_{2}}\right)\left(A_{i}\right) & =\sigma_{p_{1}}\left[\sigma_{p_{2}}\left(A_{i}\right)\right]=\sigma_{p_{1}}\left[2^{p_{2}(i)-i} \cdot A_{p_{2}(i)}\right] \\
& =2^{p_{2}(i)-i} \cdot \sigma_{p_{1}}\left(A_{p_{2}(i)}\right)=2^{p_{2}(i)-i} \cdot 2^{p_{1}\left(p_{2}(i)\right)-p_{2}(i)} \cdot A_{p_{1}\left(p_{2}(i)\right)} \\
& =2^{p_{1} \circ p_{2}(i)-i} \cdot A_{p_{1} \circ p_{2}(i)}=\sigma_{p_{1} \circ p_{2}}\left(A_{i}\right) .
\end{aligned}
$$

Since the restriction of both $\sigma_{p_{1}} \circ \sigma_{p_{2}}$ and $\sigma_{p_{1} \circ p_{2}}$ on the interval $A_{i}$ are linear, 1-1 maps onto the interval $2^{p_{1} \circ p_{2}(i)-i} \cdot A_{p_{1} \circ p_{2}(i)}$ which map the left hand end-point of $A_{i}$ to that of $2^{p_{1} \circ p_{2}(i)-i} \cdot A_{p_{1} \circ p_{2}(i)}$, it follows that $\left.\sigma_{p_{1}} \circ \sigma_{p_{2}}\right|_{A_{i}}$ and $\left.\sigma_{p_{1} \circ p_{2}}\right|_{A_{i}}$ are identical.

Now since the maps $\sigma_{p}$ and their compositions $\sigma_{p_{1}} \circ \sigma_{p_{2}}$ for any $p, p_{1}$ and $p_{2}$ in $S_{k}$ are 2-homogeneous functions, each map is totally determined by its action on $\left\{A_{i}: i=1, \ldots, k\right\} \cup\{C\}$. Therefore $\sigma_{p_{1}} \circ \sigma_{p_{2}}=\sigma_{p_{1} \circ p_{2}}$.

Let $\mathcal{H}: S_{k} \rightarrow\left\{\sigma_{p}: p \in S_{k}\right\}$ be defined as $\mathcal{H}(p)=\sigma_{p}$. Then clearly $\mathcal{H}$ is a bijection. We only need to check that $\mathcal{H}$ is a homomorphism. Indeed we have $\mathcal{H}\left(p_{1} \circ p_{2}\right)=\sigma_{p_{1} \circ p_{2}}=\sigma_{p_{1}} \circ \sigma_{p_{2}}=\mathcal{H}\left(p_{1}\right) \circ \mathcal{H}\left(p_{2}\right)$. This ends the proof.

Corollary 5. For any finite group $G$, there exists an interpolation family $\mathcal{E}_{G}$ of wavelet sets based at some $E_{0} \in \mathcal{E}_{G}$, such that $\left\{\sigma_{E_{0}}^{E}: E \in \mathcal{E}_{G}\right\}$ is isomorphic to the group $G$.

Proof. Recall that Cayley's Theorem states that any group $G$ is isomorphic to a subgroup of Sym $G$. In particular any finite group $G$ of order $k$ is isomorphic to a subgroup of $S_{k}$. Thus we only need to consider arbitrary subgroups $G$ of the group $S_{k}$ for some integer $k$. With the same sets $A$ and $A_{i}$ as in the proof of Theorem 2, let

$$
A_{g}:=\bigcup_{i=1}^{k} 2^{g(i)-i} A_{g(i)}
$$

for each $g \in G \subset S_{k}$ as before and $W_{g}:=C \cup A_{g}$ for each $g \in G \subset S_{k}$. Denote $\mathcal{E}_{G}:=\left\{W_{g}: g \in G \subset S_{k}\right\}$ and $\sigma_{g}:=\sigma_{W_{i d}}^{W_{g}}$. We will prove that $\left\{\sigma_{g}: g \in G\right\}$ is isomorphic to the group $G \subset S_{k}$. Then it will follow that $\mathcal{E}_{G}$ is the desired interpolation family of wavelet sets based at $E_{0}=W_{i d}$.

Let $\mathcal{K}: G \subset S_{k} \rightarrow\left\{\sigma_{g}: g \in G \subset S_{k}\right\}$ be defined as $\mathcal{K}(g)=\sigma_{g}$. Clearly $\mathcal{K}$ is a bijection. $\mathcal{K}$ is also a homomorphism since it is the restriction of the map $\mathcal{H}$ in the proof of Theorem 2 to the subgroup $G$. Hence $\mathcal{K}$ is an isomorphism.

\section{ACKNOWLEDGMEnTs}

The author would like to thank Dr. Larson for his guidance and encouragement without which this work would have been impossible.

\section{REFERENCES}

[C] C. K. Chui, An Introduction to Wavelets, Acad. Press, New York, 1993. MR 93f:42055

[DL] X. Dai and D. Larson, Wandering vectors for unitary systems and orthogonal wavelets, Memoirs of the AMS, July 1998, Volume 134, Number 640. MR 98m:47067

[FW] X. Fang and X. Wang, Construction of minimally supported frequency (MSF) wavelets, J. Fourier Analysis and Applications 2(1996), 315-327. MR 97d:42030

[H] P. Halmos, A Hilbert Space Problem Book, second ed., Springer-Verlag, New York, 1982. MR 84e:47001 
[HWW1] E. Hernandez, X. Wang and G. Weiss, Smoothing minimally supported (MSF) wavelets: Part I, J. Fourier Analysis and Applications, 2(1996), 329-340. MR 97h:42015

[HWW2] E. Hernandez, X. Wang and G. Weiss, Smoothing minimally supported (MSF) wavelets: Part II, to appear. MR 98b:42049

[S] D. Speegle, The s-elementary wavelets are path-connected, Proc. Amer. Math. Soc., to appear. MR 99b:42045

Department of Mathematics, The University of North Carolina at Charlotte, CharLOtTe, North Carolina 28223

E-mail address: qgu@math.uncc.edu

Current address: Department of Mathematics, Beijing University, Beijing 100871, People's Republic of China 